\title{
Impact of Non-synchronous Diagnostic Ureteroscopy on Intravesical Recurrence in Patients Underwent Radical Nephroureterectomy for Upper Tract Urothelial Carcinoma: A Propensity-Score Matched Analysis
}

\section{CURRENT STATUS: POSTED}

$\$$ Research Square

Huamao Ye

Changhai Hospital

Xiang Feng

Changhai Hospital

Yang Wang

Changhai Hospital

Rui Chen

Changhai Hospital

Meimian Hua

Changhai Hospital

Xiaojun Lu

Changhai Hospital

Xiaoqin Jiang

Changhai Hospital

Yu Fang

Changhai Hospital

Zhenjie Wu

Changzheng Hospital

Qing Yang

Changhai Hospital

Bo Yang 


\section{Changhai Hospital}

\section{Chen Lü}

Changhai Hospital

$\checkmark$ chenlouie@live.comCorresponding Author

ORCiD: https://orcid.org/0000-0001-6390-7636

Linhui Wang

Changzheng Hospital

\section{DOI:}

10.21203/rs.2.16696/v2

\section{SUBJECT AREAS}

\section{Urology \& Nephrology}

\section{KEYWORDS}

diagnostic ureteroscopy, intravesical recurrence, upper tract urothelial carcinoma, radical nephroureterectomy 


\section{Abstract}

The authors have withdrawn this preprint from Research Square 